\title{
The Combined Influence of the Local Environment and Regional Enrichment on Bird Species Richness
}

\author{
Ethan P. White ${ }^{1, *}$ and Allen H. Hurlbert ${ }^{2,3,} \quad 7 \quad+$
}

${ }^{1}$ Department of Biology and the Ecology Center, Utah State University, Logan, UT 84322

${ }^{2}$ Department of Biology, University of North Carolina at Chapel Hill, Chapel Hill, NC, 27599

${ }^{3}$ National Center for Ecological Analysis and Synthesis, Santa Barbara, CA 93101

*E-mail: epwhite@biology.usu.edu

† E-mail: hurlbert@bio.unc.edu

Keywords: biodiversity, enrichment, local environment, macroecology, regional richness, species richness.

Elements to appear in expanded online edition:

Online Appendix A. Supplemental Methods and Results

Figures to appear in color:

Figure 1, Figure 2, Figure 3

Article type: E-note 
ABSTRACT: It is generally accepted that local species richness at a site reflects the combined influence of local and regional processes. However, most empirical studies evaluate the influence of either local environmental variables or regional enrichment but not both simultaneously. Here we demonstrate the importance of combining these processes to understand continental scale richness patterns in breeding birds. We show that neither regional enrichment nor the local environment in isolation is sufficient to characterize observed patterns of species richness. Combining both sets of variables into a single model results in improved model fit and the removal of residual spatial autocorrelation. At short time scales local processes are most important for determining local richness, but as the time scale of analysis increases regional enrichment becomes increasingly important. These results emphasize the need for increased integration of multiple scales of processes into models of species richness. 
Ecologists have long debated whether the richness of local communities is limited primarily by local factors such as the availability of niches (MacArthur 1964; Tilman 2004) or resources (Brown 1981; Wright 1983), or alternatively whether local richness is determined by the richness of the regional pool and thus more strongly related to regional scale variables that reflect evolutionary history and colonization dynamics (Ricklefs 1987; Ricklefs 2007). Local processes hypothesized to limit richness include competition for limited resources (Brown 1981), limited niche space (MacArthur 1964), and limiting similarity (Tilman 2004), all of which make it more difficult for species to persist at sites where large numbers of species already occur. An alternative explanation, based on the observation that local sites do not appear to be saturated with species (Ricklefs 1987; Stohlgren et al. 2008), suggests that local species richness is actually limited by the availability of species to colonize the site from the regional pool (Cornell and Lawton 1992; Harrison and Cornell 2008). While the specifics of exactly how regional richness translates into local richness have been poorly explored, it has been proposed that this may happen through a simple, neutral, colonization-extinction equilibrium (He et al. 2005). Under this scenario, local richness is expected to be more strongly tied to processes and events operating beyond the scale of the local community, such as diversification rates and biogeographic history (Ricklefs 1987; Ricklefs 2007).

Interestingly, most analyses of local scale species richness patterns analyze either the effects of the local environment (e.g., Gough et al. 2000; Kaspari et al. 2000) or the effects of regional richness (e.g., Karlson et al. 2004; Witman et al. 2004), despite suggestions that both sets of processes are important (Kaspari et al. 2000; Ricklefs 2000; Harrison and Cornell 2008). Only a small fraction of richness studies have actually examined the joint influence of local and regional factors on local species richness (e.g., Angermeier and Winston 1998; Griffiths 1999; Freestone 
and Harrison 2006; Harrison et al. 2006; Qian et al. 2007; Hortal et al. 2008), leading to calls for greater integration of local and regional influences into richness models (Harrison and Cornell 2008). Furthermore, in studies where both local environmental factors and regional richness have been included in a single analysis, the analyses are typically used to confirm that both processes are operating or to argue that one of the processes is important even after controlling for the other (Cornell and Karlson 1996; Angermeier and Winston 1998; Karlson and Cornell 1999; Freestone and Harrison 2006). This is an important step towards integrating the contributions of the two scales of process, but stops short of providing information about the relative importance of local and regional influences and how they interact to determine local diversity.

Determining the relative importance of local factors and regional enrichment represents an important step towards a general understanding of the processes governing species richness (Angermeier and Winston 1998; Griffiths 1999; Ricklefs 2000). If either the local environment or regional enrichment dominates observed richness patterns then processes operating at that scale will be the key to understanding diversity. However, if both scales contribute substantially to observed richness patterns, then it may be necessary to explicitly consider both environmental contributions and regional enrichment in models and empirical studies of species richness (Harrison et al. 2006; Harrison and Cornell 2008). Variance partitioning provides a tool for ascertaining the relative contributions of these two scales of processes. It determines how much of the overall variance in local species richness can be uniquely ascribed to local environmental variables and regional richness, and how much of the variance is explained by some combination of the two categories of variables, but cannot be uniquely ascribed to either (Legendre and Legendre 1998). As such it provides a valuable, but underutilized tool for understanding the determinants of species richness. A pair of recent papers has partitioned the explained variance 
between a categorical region factor and the local environment (Qian et al. 2007; Hortal et al. 2008), but we know of only one study to have used variance partitioning to evaluate the relative contributions of regional enrichment and local environmental variables (Harrison et al. 2006).

Here we show that for North American breeding birds both the local environment and regional enrichment contribute substantially to observed variation in species richness and use variance partitioning to the explore the relative importance of these two scales of processes. We start by establishing that both local environmental variables and regional richness are correlated with local richness and show that looking at only one of these categories of variables misses significant patterns in local richness. Variance partitioning analyses indicate approximately equal contributions of the two sets of variables at longer time scales (i.e., 10 years). Further, we establish the presence of collinearity between the two categories of predictor variables and illustrate its influence on conclusions drawn from analyzing only one category at time. Finally, we evaluate the influence of the time-scale of analysis on the variance partitioning results, and use the information gained from this analysis to explore possible ways in which local and regional influences combine to govern local species richness.

\section{Methods}

Data on local scale species richness were taken from the North American Breeding Bird Survey (Sauer et al. 2007)(BBS; Sauer et al. 2007). The BBS is a coordinated continental scale survey of bird diversity and abundance conducted once each year during the breeding season, typically in June. Each survey is conducted along a $40 \mathrm{~km}$ route, with stops every $800 \mathrm{~m}$. At each stop a single observer conducts a three minute point count where all detected individuals are identified to species and tallied. Groups not well sampled using BBS methods including water birds, nocturnal birds, and raptors were excluded from analyses. As such, we focus on 305 land 
bird species for analysis. In addition we only used routes that were judged to be quality routes by the BBS coordinator (i.e., run type $=1$ ). To minimize the potential underestimation of local richness due to sampling effects, we calculated the average number of species observed over all possible five year windows from 1997-2006 (see McGill 2003). While it has been suggested that observed values of species richness may be biased due to differences in detection probabilities (Nichols et al. 1998), the methods proposed for addressing this potential bias in BBS data are based on unrealistic assumptions regarding the homogeneity of BBS routes (e.g., see Boulinier et al. 1998) and analyses evaluating broad scale richness patterns based on observed richness and estimated richness find almost identical results (Evans et al. 2008). Therefore we utilize observed species richness values in this study.

For each of the 871 routes surveyed every year during this time span (Appendix A) we characterized six local scale environmental variables within a $40 \mathrm{~km}$ radius of the route's starting coordinates, a scale that ensures the inclusion of the entire route. Data on mean summer (June-August) and winter (December-February) temperatures and annual precipitation were obtained from the Climatic Research Unit (http://www.cru.uea.ac.uk/cru/data/tmc.htm), and are long term averages from 1961-1990 at 10’ resolution. Summer and winter values of the normalized difference vegetation index (NDVI; a remotely sensed measure of greenness) were derived from the NASA Pathfinder AVHRR satellite at $8 \mathrm{~km}$ base resolution (average values from 1982-2000 excluding 1994). NDVI is well correlated with measures of productivity and standing green biomass (Chong et al. 1993; Paruelo et al. 1997) and is used here as a measure of productivity. We characterized the mean elevation of each site using a 30-arc second digital elevation model of North America (http://edc.usgs.gov/products/elevation/gtopo30/README.html). Mean elevation is highly 
correlated with a number of measures of elevational heterogeneity and was chosen out of these measures because it yields the strongest correlations with richness. This suite of environmental variables was chosen because the data are readily available at local grains and continental extents and because measures of productivity (including NDVI) are known to be the primary correlates of species richness in bird communities evaluated at this combination of grain and extent in North America (Hurlbert and Haskell 2003; Currie et al. 2004) and at continental extents in general (Hawkins et al. 2003a; Hawkins et al. 2003b). We used long-term average data to facilitate the cross time-scale analyses. Due to issues with data availability, the temporal spans of the biological data and environmental data overlap only partially or not at all. However, any differences from the long term averages will be small compared to the continental variation in these variables which is of interest.

The richness of the regional species pool for each BBS survey was determined by overlaying the range maps for all species included in the study, and counting the number of maps that overlapped each local survey location. Range map data represent independent assessments of species distributions based on expert opinion and were provided by NatureServe (Ridgely et al. 2000). This approach could potentially include species in the pool that cannot use the particular habitat present at the site (Srivastava 1999), but we follow Ricklefs’ (2000) recommendation that this is the most appropriate approach to defining the regional pool.

Three types of regression models were used to explain variation in local species richness. In the Local Environment model all six local environmental variables were used as predictors. In the Regional Enrichment model, regional richness was the sole predictor. The Combined model included both sets of predictor variables. In all models, quadratic terms were included for each variable to account for potential non-linear relationships. Half of the routes were randomly 
selected to fit the regression models and the other half of the routes were used to evaluate their performance. Variance partitioning analyses were conducted using standard methods based on the results of these three regression models (Legendre and Legendre 1998). Because values of species richness are reasonably large we treat them as continuous data (as is common practice; e.g., Hawkins et al. 2003a; Rahbek et al. 2007) instead of explicitly incorporating discrete error structure (as is also commonly done e.g., Schweiger et al. 2007). The performance of the three models was compared using $\mathrm{AIC}_{c}$ (Burnham and Anderson 2002).

In addition to the multiple regressions we conducted a path analysis to examine the causal relationships between these factors and the regional environment, which we defined as the same suite of environmental variables measured within a $320 \mathrm{~km}$ radius of the starting coordinates. Results were qualitatively similar using regional scales with radii ranging from 160-640 km. We also evaluated patterns of spatial autocorrelation in the residuals of the models using Moran’s I (Legendre and Legendre 1998). In calculating Moran’s I, we grouped all pairwise comparisons of BBS routes into 25 distance classes, each containing the same number of comparisons. The confidence intervals are based on a Bonferroni-corrected significance level of 0.002 .

We also evaluated the effect of time-scale on the variance partitioning analysis by using windows from 1 to 10 years to estimate species richness. For each time scale we calculated the mean cumulative local richness of all possible contiguous samples of the appropriate length occurring between 1997 and 2006 (see White 2004). These mean richness values were then analyzed as described above for each different time-span. The ten year time span represents a compromise between having a long enough time span to evaluate time scale effects and having enough continuously sampled routes to provide a good coverage of the sampled regions.

\section{Results and Discussion}




\section{Analyzing local and regional determinants separately and simultaneously}

Within this single dataset we observe two common patterns of species richness: 1) a positive relationship between local and regional richness (fig. 1a; $r=0.56$ ); and 2) a strong relationship between a suite of local environmental variables and local richness, where the primary correlation is with an estimate of productivity (fig. 1c; $r_{\mathrm{NDVI}, \text { summer }}=0.68$ ). The majority of studies investigating patterns of species richness examine only one of these two types of relationships, and the presence of a strong correlation has been taken (implicitly or explicitly) as evidence for that variable or suite of variables as an important determinant of observed geographic patterns (e.g., Kaspari et al. 2000; Allen et al. 2002; Karlson et al. 2004; Witman et al. 2004). However, when we look at either pattern in more detail we see a significant signal of the other set of variables on species richness. The simple regional richness model significantly underestimates species richness in communities with high NDVI and overestimates richness in communities with low NDVI (fig. 1a-b). The equivalent pattern is seen in the local environment model, which underestimates richness when the regional pool is species rich, and overestimates it where the regional pool is species poor (fig. 1c-d). Because both sets of variables have an important influence on local richness, including both improves the overall fit of the model (fig. 1e-f), with the combined model clearly favored in AIC comparisons ( $\Delta \mathrm{AIC}_{c}$ for the Local Environment model $=162.0 ;$ Regional Enrichment model $=290.3 ;$ Combined model $=0 ; \Delta \mathrm{AIC}_{c}$ values $>10$ are considered to represent almost no support for the model). These results are similar to those of Harrison et al. (2006), which indicate that both local environmental factors and regional richness influence the local richness of serpentine floras in California. However, in contrast to Harrison et al.'s results (see also Harrison and Cornell 2008) our analysis suggests that productivity is directly related to richness at the local scale (fig. 1; Appendix A). 
If the contributions of local environment and regional richness were independent of one another then while a combined analysis would produce a model with greater explanatory power, conclusions regarding the importance of either factor based on analyzing it in isolation would be unaffected. However, our results suggest collinearity between regional richness and the local environmental variables (all environmental variables are correlated with regional richness; P-values $<0.005$ ) leading to a substantial fraction of variation that cannot be uniquely ascribed to either local or regional processes (fig. 1f). Had we analyzed either the local environment or regional richness alone, we would have ascribed this non-unique variance to the variables we chose to investigate. In the case of regional richness, its unique importance would have been overestimated by over 100\%. In general, studies examining either the local environment or regional richness in isolation risk overemphasizing the importance of the chosen predictors due to the underlying covariance with unconsidered predictor variables. While this is a general problem in regression analyses, it is particularly relevant here because there are many reasons to think that regional richness might frequently co-vary with local environmental variables (Harrison et al. 2006; Harrison and Cornell 2008). The collinearity between regional richness and local environmental variables also has consequences for the interpretation of individual predictors on local richness (Graham 2003). For example, the coefficient for regional richness decreases by 70\% when local environmental variables are added to the model (Appendix A). Such differences will be crucial when attempting to evaluate theories that make specific predictions about model parameters (e.g., Allen et al. 2002; Algar et al. 2007; Hawkins et al. 2007), because if the contribution of regional enrichment is not controlled for then the wrong estimate of the fitted parameter may be compared to the theoretical value.

In addition to yielding improvements in overall model fit and parameter estimation, the 
combined model substantially decreases the spatial structure of model residuals. The raw richness values exhibit significant spatial autocorrelation at distances up to $1500 \mathrm{~km}$ (fig. 2), which could present a problem for modeling the data using non-spatial methods (Lennon 2000). The residuals of the regional enrichment model display similar spatial autocorrelation (fig. 2). Modeling richness with local environmental data reduces autocorrelation in the residuals (see also Hurlbert and White 2005; Hortal et al. 2008), although smaller scale positive autocorrelation remains in the shortest distance class. The model combining local environmental factors and regional richness eliminates this remaining residual autocorrelation resulting in no significant autocorrelation at any scale (fig. 2). This suggests that the residual spatial structure in some local scale species richness models may be due to the enrichment of local communities by the regional species pool, potentially reducing the need for spatial regression techniques when richness is modeled using a combined approach (Diniz-Filho et al. 2003).

While the inclusion of both regional richness and local environmental variables in a single analysis of species richness is relatively rare (Harrison and Cornell 2008), it is more common to see different scales of environmental variables evaluated in the same analysis (e.g., Rahbek and Graves 2001; Hurlbert and Haskell 2003). More sophisticated analyses using structural equation modeling will be required to tease apart the details of how regional and local environments influence regional and local richness and the effect of regional richness on local richness. That said our results are qualitatively similar when using different scales of environmental variables and our simple path analysis supports our general conclusions (Appendix A). Spatial scale, autocorrelation, and "pseudoreplication" Analyses similar to ours have been criticized as being pseudoreplicated due to the spatially autocorrelated nature of the regional species pool (Srivastava 1999). For example, in this study 
the median percentage of shared species among all pairwise regional pool comparisons was 32\%. However, the regional species pool is no different than any other spatially autocorrelated predictor variable. For example, at the scale at which birds perceive elevational differences most of the Midwest is a single elevational "region". This autocorrelation results in an overestimate of the number of degrees of freedom if autocorrelation persists in the model residuals (e.g., Lichstein et al. 2002). To be clear, the pseudoreplication described by Srivastava (1999) in this observational context is simply spatial autocorrelation. Because our central analysis is that of the combined model and the combined model successfully removes spatial autocorrelation from the residuals, our analysis does not overestimate the degrees of freedom and thus yields valid statistical results (Lichstein et al. 2002; Diniz-Filho et al. 2003; Rangel et al. 2006). Srivastava (1999) recommends that in order to avoid pseudoreplication, local richness values should be averaged to produce only a single value for each broadly defined geographic region. Our analyses support the idea that models based solely on regional richness will exhibit strong autocorrelation (fig. 2) and therefore support Srivastava’s (1999) concern with respect to non-combined analyses as described above. However, her proposed solution of averaging data points within regions eliminates all within-region variability in local richness, and is thus inappropriate for assessing the relative importance of the local environment which may be important in driving that variability. Studies of local-regional relationships that remove local scale variability by averaging local richness values within a larger region (e.g., Srivastava 1999, Karlson et al. 2004) ignore this meaningful variation and may exaggerate the importance of the regional pool. Our combined model solves this problem by successfully modeling the observed autocorrelation as being driven by meaningful environmental variation and regional enrichment and thus allows individual local scale data points to be incorporated without statistical 
complications. More sophisticated spatially explicit modeling may well provide key additional insights, but the current approach represents a valuable first step towards understanding these patterns.

Temporal scale and the relative importance of local and regional processes

We explored the influence of the temporal resolution used to characterize local species richness and found that increasing the time-scale has only a small influence on the overall predictive power of the regression model (fig. 3). However, the proportions of the variance explained by the local environment and regional enrichment changed as the time-scale increased (fig. 3). While local environmental variables largely dominate at one-year timescales, at decadal timescales, local and regional variables explain similar amounts of variance in local richness. The processes governing species richness are expected to change with the scale of analysis due to changes in the physical and biological processes dominating at different scales (Holling 1992). For example, species interactions occur over days and hectares whereas speciation dynamics occur over regional to continental extents and geological time periods. As such, it makes sense that regional processes play an increasing role at longer time scales. These results confirm the suggestion that temporal scale should affect the strength, as well as the shape, of the local-regional richness relationship (Srivastava 1999).

The changes in explained variance result from changes in cumulative local richness with time-scale. The accumulation of species occurs due to both increased sampling intensity, whereby rare species that were present at the site are finally sampled, and real turnover, where species that were not present in a given year colonize the site (White 2004, White et al. 2006). Species in this latter group include both vagrants and species invading the site in response to changes in environmental conditions. Thus, the species richness of a local site over a broader 
temporal window increasingly reflects temporal beta diversity relative to single year alpha diversity, and it has been shown that beyond time-scales of $\sim 2-3$ years that this beta diversity is driven more by ecological processes than sampling intensity (White 2004). As such, our results suggest that local environmental constraints are most important for determining alpha diversity in North American bird communities, while regional enrichment is important for explaining patterns of temporal beta diversity. In the face of environmental variability, richer regional species pools are more likely to contain species that can successfully cope with novel conditions. In addition, even in the absence of environmental change, richer species pools are expected to contribute more species to local communities via mass effects (sensu Shmida and Wilson 1985).

This suggests a possible avenue for integrating local and regional influences on species richness. It has been proposed that local communities are actually composed of two potentially discrete groups of species: 1) core (or source) species, which maintain viable populations at a site because they are well suited to the local ecological conditions; and 2) occasional (or sink or vagrant) species that periodically occur at the site through random colonization events, but fail to persist in the system (MacArthur 1960; Magurran and Henderson 2003; Belmaker 2009). Since a site will typically contain some occasional species, this would explain the unique contribution of regional enrichment, even at the shortest time-scales. If this hypothesis is valid then observed regional influences may occur via mass effects with the number of core species limited by local environmental conditions and the number of occasional species influenced by the richness of the regional pool. The ability of local environmental variables to explain one-year richness values may thus reflect the constraints imposed by the local environment that limit the number of core species which can maintain stable populations at that site. Vagrant species would not be expected to be restricted by this limited capacity as only a few dispersing individuals are encountered 
(MacArthur 1960; Magurran and Henderson 2003; Belmaker 2009). The fact that regional richness becomes increasingly important for understanding local richness over longer temporal windows reflects the fact that the number of species that might ephemerally colonize a site should increase with the time period of sampling (Grinnell 1922) and the size of the species pool. Thus occasional species richness should be driven by regional enrichment and should be more prevalent at longer time scales. Admittedly this clear distinction between core and occasional species is overly simplistic. For example, the status of species can change through time in response to changing environmental conditions or biotic interactions (Brown et al. 2001). In addition, distinguishing between core and occasional species will often be difficult and might require choosing an arbitrary cutoff based on abundance or persistence (e.g., Magurran and Henderson 2003).

Finally, it is also worth noting that as time scale increases there is an increase in the proportion of variation that cannot be uniquely ascribed to either local or regional influences, and thus, the relative contribution of these processes becomes less distinguishable (fig. 3). As a result analyses that use local richness measures based on longer time scales, e.g. regional floral and faunal lists, risk overestimating the importance of either local or regional processes if both are not examined simultaneously using this type of variance partitioning framework.

\section{Acknowledgements}

We thank Susan Harrison, Mevin Hooten, Xiao Xiao, Andrew Solow, Mark McPeek and three anonymous reviewers for helpful comments on this research and/or manuscript. We acknowledge the efforts of U.S. and Canadian BBS participants in the field, as well as USGS and CWS researchers and managers, and thank NatureServe, Robert Ridgely, James Zook, The Nature Conservancy - Migratory Bird Program, Conservation International - CABS, World 
Wildlife Fund, and Environment Canada - WILDSPACE for providing the range map data. AHH was supported by the National Center for Ecological Analysis and Synthesis, a Center funded by NSF (DEB-0072909), and the University of California Santa Barbara. EPW was supported by a Postdoctoral Fellowship in Biological Informatics from NSF (DBI-0532847). 


\section{Online Appendix A. Supplemental Methods and Results.}

\section{Path analysis - detailed methods and results}

We conducted a simple path analysis using least squares methods to explore the manner in which regional scale climate influenced local scale richness. For simplicity we: 1) only analyzed the single most important climate variable (summer NDVI; univariate correlations were equivalent for NDVI and NDVI ${ }^{2}$ so we chose the linear form for direct comparison with Harrison et al. 2006); 2) used only the most important transformation of regional richness (the untransformed data); and 3) used all of the data instead of separating it into training and validation sets. The results (Figure S2) indicate that most of the contribution of regional scale NDVI (defined as the average NDVI within a $320 \mathrm{~km}$ radius circle of the starting location of the route) on local richness was through its influence on local NDVI and local NDVI's influence on richness (0.46). The influence of regional NDVI though regional richness was relatively minor (0.12). This result is contrary to that of Harrison et al. (2006; see also Harrison and Cornell 2008) who show that for serpentine plant communities in CA that regional NDVI's influence occurs primarily through its influence on regional richness. 
Table S1. Parameter estimates and multivariate statistical results for the three statistical models.

MODEL AND PARAMETER

Regional Enrichment

RegRich

RegRich $^{2}$

Local Environment

SumTemp

SumTemp ${ }^{2}$

WinTemp

WinTemp ${ }^{2}$

AnnPrecip

AnnPrecip ${ }^{2}$

SumNDVI

SumNDVI ${ }^{2}$

WinNDVI

WinNDVI ${ }^{2}$

Elev

Elev $^{2}$

Combined Model
RegRich
RegRich $^{2}$
SumTemp
SumTemp ${ }^{2}$
WinTemp
WinTemp ${ }^{2}$
AnnPrecip
AnnPrecip ${ }^{2}$
SumNDVI
SumNDVI ${ }^{2}$
WinNDVI
WinNDVI ${ }^{2}$
Elev
Elev $^{2}$

PARAMETER ESTIMATE [95\% CI]

$$
1.21[0.73,1.69]
$$$$
-0.004 \text { [-0.007, -0.001] }
$$

P-VALUE

MODEL $R^{2}$

0.331

$<10^{-6}$

0.007

$\begin{array}{cc}2.11[-0.03,4.24] & 0.054 \\ -0.05[-0.103,0.005] & 0.078 \\ -0.14[-0.57,0.29] & 0.517 \\ -0.04[-0.05,-0.02] & <10^{-4} \\ 0.003[-0.008,0.014] & 0.568 \\ -4.70 \mathrm{e}-6[-8.35 \mathrm{e}-6,-1.06 \mathrm{e}-6] & 0.012 \\ 15.6[-29.5,60.8] & 0.498 \\ 55.7[13.4,98.0] & 0.010 \\ 42.8[14.5,71.2] & 0.003 \\ -60.8[-100.1,-20.8] & 0.003 \\ -0.008[-0.015,-0.002] & 0.014 \\ 3.08 \mathrm{e}-6[7.60 \mathrm{e}-7,5.40 \mathrm{e}-6] & 0.010\end{array}$

$\begin{array}{cc}0.36[-0.17,0.90] & 0.184 \\ 6.02 \mathrm{e}-4[-2.57 \mathrm{e}-3,3.78 \mathrm{e}-3] & 0.710 \\ -0.84[-2.96,1.27] & 0.435 \\ 0.03[-0.02,0.09] & 0.248 \\ -0.10[-0.51,0.31] & 0.630 \\ -0.02[-0.04,0.002] & 0.085 \\ -4.13 \mathrm{e}-4[-0.01,0.01] & 0.938 \\ -1.70 \mathrm{e}-6[-5.19 \mathrm{e}-6,1.78 \mathrm{e}-6] & 0.338 \\ 77.8[33.1,122.5] & 0.001 \\ -29.0[-73.5,15.4] & 0.201 \\ 34.2[6.81,61.5] & 0.015 \\ -42.2[-82.4,-2.00] & 0.040 \\ -6.65 \mathrm{e}-3[-1.27,-4.21 \mathrm{e}-4] & 0.015 \\ 6.58 \mathrm{e}-7[-1.57 \mathrm{e}-6,2.88 \mathrm{e}-6] & 0.563\end{array}$




\section{Literature Cited}

Algar, A. C., J. T. Kerr, and D. J. Currie. 2007. A test of Metabolic Theory as the mechanism underlying broad-scale species-richness gradients. Global Ecology and Biogeography 16:170-178.

Allen, A. P., J. H. Brown, and J. F. Gillooly. 2002. Global biodiversity, biochemical kinetics, and the energetic-equivalence rule. Science 297:1545-1548.

Angermeier, P. L., and M. R. Winston. 1998. Local vs. regional influences on local diversity in stream fish communities of Virginia. Ecology 79:911-927.

Belmaker, J. 2009. Species richness of resident and transient coral-dwelling fish responds differentially to regional diversity. Global Ecology and Biogeography 18:426-436.

Boulinier, T., J. D. Nichols, J. R. Sauer, J. E. Hines, and K. H. Pollock. 1998. Estimating species richness: the importance of heterogeneity in species detectability. Ecology 79:1018-1028.

Brown, J. H. 1981. 2 decades of homage to Santa-Rosalia: toward a general theory of diversity. American Zoologist 21:877-888.

Brown, J. H., T. G. Whitham, S. K. M. Ernest, and C. A. Gehring. 2001. Complex Species Interactions and the Dynamics of Ecological Systems: Long-Term Experiments. Science 293:643-650.

Burnham, K. P., and D. R. Anderson. 2002, Model selection and multimodel inference: a practical information-theoretic approach. New York, Springer.

Chong, D. L. S., E. Mougin, and J. P. Gastellu-Etchegorry. 1993. Relating the global vegetation index to net primary productivity and actual evapotranspiration over Africa. International Journal of Remote Sensing 14:1517 - 1546. 
Cornell, H. V., and R. H. Karlson. 1996. Species richness of reef-building corals determined by local and regional processes. Journal of Animal Ecology 65:233-241.

Cornell, H. V., and J. H. Lawton. 1992. Species interactions, local and regional processes, and limits to the richness of ecological communities - a theoretical perspective. Journal of Animal Ecology 61:1-12.

Currie, D. J., G. G. Mittelbach, H. V. Cornell, R. Field, J. F. Guegan, B. A. Hawkins, D. M. Kaufman et al. 2004. Predictions and tests of climate-based hypotheses of broad-scale variation in taxonomic richness. Ecology Letters 7:1121-1134.

Diniz-Filho, J. A., L. M. Bini, and B. A. Hawkins. 2003. Spatial autocorrelation and red herrings in geographical ecology. Global Ecology And Biogeography 12:53-64.

Evans, K. L., S. E. Newson, D. Storch, J. J. D. Greenwood, and K. J. Gaston. 2008. Spatial scale, abundance and the species-energy relationship in British birds. Journal of Animal Ecology 77:395-405.

Freestone, A. L., and S. Harrison. 2006. Regional enrichment of local assemblages is robust to variation in local productivity, abiotic gradients, and heterogeneity. Ecology Letters 9:95-102.

Gough, L., C. W. Osenberg, K. L. Gross, and S. L. Collins. 2000. Fertilization effects on species density and primary productivity in herbaceous plant communities. Oikos 89:428-439.

Graham, M. H. 2003. Confronting multicollinearity in ecological multiple regression. Ecology 84:2809-2815.

Griffiths, D. 1999. On investigating local-regional species richness relationships. Journal of Animal Ecology 68:1051-1055.

Grinnell, J. 1922. The role of the "accidental". Auk 39:373-380. 
Hanski, I. 1982. Dynamics of regional distribution: the core and satellite species hypothesis. Oikos 38:210-221.

Harrison, S., and H. Cornell. 2008. Toward a better understanding of the regional causes of local community richness. Ecology Letters 11:969-979.

Harrison, S., H. D. Safford, J. B. Grace, J. H. Viers, and K. F. Davies. 2006. Regional and local species richness in an insular environment: Serpentine plants in California. Ecological Monographs 76:41-56.

Hawkins, B., R. Field, H. Cornell, D. Currie, J. Guegan, D. Kaufman, J. Kerr et al. 2003a. Energy, water, and broad-scale geographic patterns of species richness. Ecology 84:3105-3117.

Hawkins, B. A., F. S. Albuquerque, M. B. Araujo, J. Beck, L. M. Bini, F. J. Cabrero-Sanudo, I. Castro-Parga et al. 2007. A global evaluation of metabolic theory as an explanation for terrestrial species richness gradients. Ecology 88:1877-1888.

Hawkins, B. A., E. E. Porter, and J. A. F. Diniz-Filho. 2003b. Productivity and history as predictors of the latitudinal diversity gradient of terrestrial birds. Ecology 84:1608-1623.

He, F. L., K. J. Gaston, E. F. Connor, and D. S. Srivastava. 2005. The local-regional relationship: Immigration, extinction, and scale. Ecology 86:360-365.

Holling, C. S. 1992. Cross-scale morphology, geometry, and dynamics of ecosystems. Ecological Monographs 62:447-502.

Hortal, J., J. Rodriguez, M. Nieto-Diaz, and J. M. Lobo. 2008. Regional and environmental effects on the species richness of mammal assemblages. Journal of Biogeography 35:1202-1214. 
Hurlbert, A. H., and J. P. Haskell. 2003. The effect of energy and seasonality on avian species richness and community composition. American Naturalist 161:83-97.

Hurlbert, A. H., and E. P. White. 2005. Disparity between range map- and survey-based analyses of species richness: patterns, processes and implications. Ecology Letters 8:319-327.

Karlson, R. H., and H. V. Cornell. 1999. Integration of local and regional perspectives on the species richness of coral assemblages. American Zoologist 39:104-112.

Karlson, R. H., H. V. Cornell, and T. P. Hughes. 2004. Coral communities are regionally enriched along an oceanic biodiversity gradient. Nature 429:867-870.

Kaspari, M., S. O'Donnell, and J. Kercher. 2000. Energy, density, and constraints to species richness: Ant assemblages along a productivity gradient. American Naturalist 155:280-293.

Legendre, P., and L. Legendre. 1998, Numerical Ecology. Amsterdam, Elsevier Science B.V.

Lennon, J. J. 2000. Red-shifts and red herrings in geographical ecology. Ecography 23:101-113.

Lichstein, J. W., T. R. Simons, S. A. Shriner, and K. E. Franzreb. 2002. Spatial autocorrelation and autoregressive models in ecology. Ecological Monographs 72:445-463.

MacArthur, R. 1960. On the relative abundance of species. American Naturalist 94:25-36.

MacArthur, R. H. 1964. Environmental factors affecting bird species diversity. American Naturalist 98:387-396.

Magurran, A., and P. Henderson. 2003. Explaining the excess of rare species in natural species abundance distributions. Nature 422:714-716.

McGill, B. 2003. A test of the unified neutral theory of biodiversity. Nature 422:881-885.

Nichols, J. D., T. Boulinier, J. E. Hines, K. H. Pollock, and J. R. Sauer. 1998. Inference methods for spatial variation in species richness and community composition when not all species are detected. Conservation Biology 12:1390-1398. 
Paruelo, J. M., H. E. Epstein, W. K. Lauenroth, and I. C. Burke. 1997. ANPP estimates from NDVI for the central grassland region of the United States. Ecology 78:953-958.

Qian, H., P. S. White, and J. S. Song. 2007. Effects of regional vs. ecological factors on plant species richness: An intercontinental analysis. Ecology 88:1440-1453.

Rahbek, C. 2005. The role of spatial scale and the perception of large-scale species-richness patterns. Ecology Letters 8:224-239.

Rahbek, C., N. J. Gotelli, R. K. Colwell, G. L. Entsminger, T. F. L. V. B. Rangel, and G. R. Graves. 2007. Predicting continental-scale patterns of bird species richness with spatially explicit models. Proceedings of the Royal Society B: Biological Sciences 274:165-174.

Rahbek, C., and G. R. Graves. 2001. Multiscale assessment of patterns of avian species richness. Proceedings of the National Academy of Sciences of the United States of America 98:4534-4539.

Rangel, T., J. A. F. Diniz-Filho, and L. M. Bini. 2006. Towards an integrated computational tool for spatial analysis in macroecology and biogeography. Global Ecology and Biogeography $15: 321-327$

Ricklefs, R. E. 1987. Community diversity - relative roles of local and regional processes. Science 235:167-171.

—. 2000. The relationship between local and regional species richness in birds of the Caribbean Basin. Journal of Animal Ecology 69:1111-1116.

—. 2007. History and diversity: Explorations at the intersection of ecology and evolution. American Naturalist 170:S56-S70. 
Sauer, J. R., J. E. Hines, and J. Fallon. 2007. The North American Breeding Bird Survey, Results and Analysis 1966-2006. Version 10.13.2007. USGS Patuxent Wildlife Research Center, Laurel, MD.

Schweiger, O., M. Musche, D. Bailey, R. Billeter, T. Diekötter, F. Hendrickx, F. Herzog et al. 2007. Functional richness of local hoverfly communities (Diptera, Syrphidae) in response to land use across temperate Europe. Oikos 116:461-472.

Srivastava, D. S. 1999. Using local-regional richness plots to test for species saturation: pitfalls and potentials. Journal of Animal Ecology 68:1-16.

Stohlgren, T. J., D. T. Barnett, C. S. Jarnevich, C. Flather, and J. Kartesz. 2008. The myth of plant species saturation. Ecology Letters 11:313-322.

Tilman, D. 2004. Niche tradeoffs, neutrality, and community structure: A stochastic theory of resource competition, invasion, and community assembly. Proceedings of the National Academy of Sciences of the United States of America 101:10854-10861.

White, E. P. 2004. Two-phase species-time relationships in North American land birds. Ecology Letters 7:329-336.

White, E. P., P. B. Adler, W. K. Lauenroth, R. A. Gill, D. Greenberg, D. M. Kaufman, A. Rassweiler et al. 2006. A comparison of the species-time relationship across ecosystems and taxonomic groups. Oikos 112:185-195.

Willig, M. R., D. M. Kaufman, and R. D. Stevens. 2003. Latitudinal gradients of biodiversity: Pattern, process, scale, and synthesis. Annual Review of Ecology Evolution and Systematics 34:273-309. 
Witman, J. D., R. J. Etter, and F. Smith. 2004. The relationship between regional and local species diversity in marine benthic communities: A global perspective. Proceedings of the National Academy of Sciences of the United States of America 101:15664-15669.

Wright, D. H. 1983. Species-energy theory: an extension of species-area theory. Oikos 41:496-506. 


\section{Figure Legends}

Figure 1: Comparison of models of local species richness (at the five-year time scale) based on regional richness, local environmental factors, and both combined. For illustrative purposes plots a-e include data for all sites with predicted values and residuals based on the models generated using the data reserved for model building. In plots b, d, and e the plus symbols indicate data used to build the model and the circles indicate data used to test the model. (a) Observed local richness as a function of predicted local richness based on the Regional Enrichment model, color coded by summer NDVI, the single best environmental predictor of local richness. Results are plotted as an interpolated surface of NDVI values (color coded as quantiles) to allow the clear presentation of large numbers of overlapping points. The solid line is the 1:1 line. (b) Residuals of the Regional Enrichment model as a function of summer NDVI. (c) Observed local richness as a function of predicted local richness based on the Local Environment model, with points color coded by regional richness (plotting details as in (a)). (d) Residuals of the Local Environment model as a function of regional richness. (e) Observed vs. predicted plot for the Combined model including both regional richness and local environmental factors. (f) Comparison of the performance of the three models based on proportion of variance explained. The Combined model's variance is partitioned to show the unique contributions of the local environment (blue), regional richness (red), and the variance that is described by some combination of the two sets of factors, but is not uniquely ascribable to either (grey). Only data reserved for model testing was used for this final analysis.

Figure 2: Patterns of spatial autocorrelation (Moran's $I$ ) at the five year time-scale for the raw richness data (black) and the residuals of the three models: Regional Enrichment (red), Local Environment (blue), and the Combined model (green). Confidence intervals (error bars) for 
Moran's I are Bonferroni corrected for the number of distance classes (i.e., error bars are equal to

$$
\pm 3.08 \times \sigma_{I}
$$

). Zero autocorrelation is shown by the dashed black line.

Figure 3: Effect of time scale on the partitioning of variance of species richness in North American breeding birds into effects of the local environment (blue), regional enrichment (red), and variance explained by some combination of the two sets of factors that cannot be uniquely ascribed to both (grey).

Figure S1. Map of routes from the Breeding Bird Survey of North America that were analyzed in this study.

Figure S2. Results of the path analysis evaluating the manner in which regional scale climate influences local scale richness. Most of the contribution of regional scale NDVI (defined as the average NDVI within a $320 \mathrm{~km}$ radius circle of the starting location of the route) on local richness was through its influence on local NDVI and local NDVI's influence on richness (0.46). The influence of regional NDVI though regional richness was relatively minor (0.12). See Online Appendix A for details. 


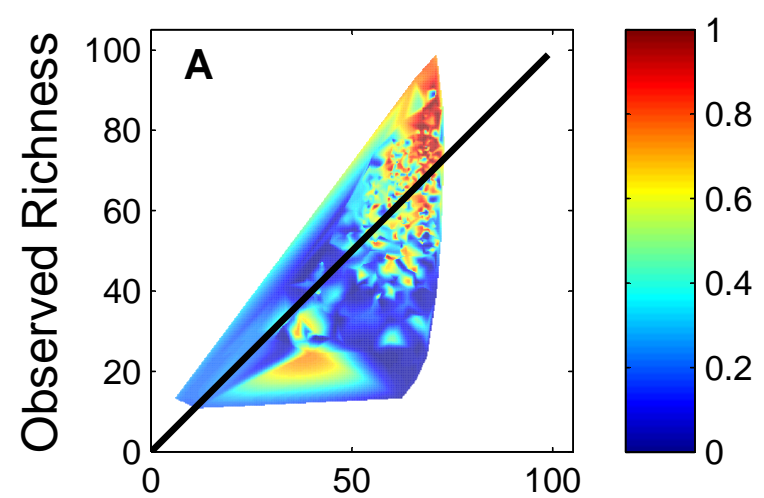

Predicted Local Richness (Regional Richness)
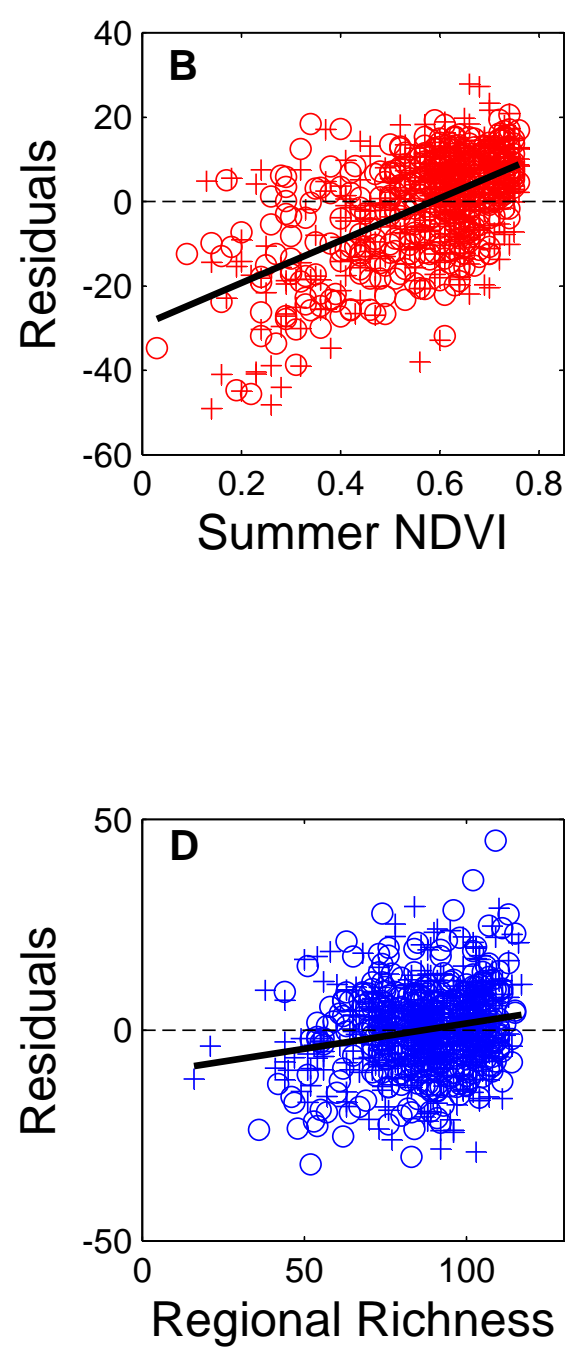

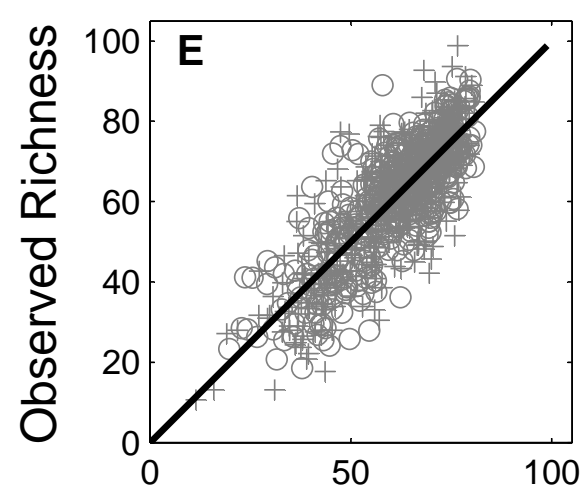

Predicted Local Richness (Loc. Env. + Reg. Rich.)

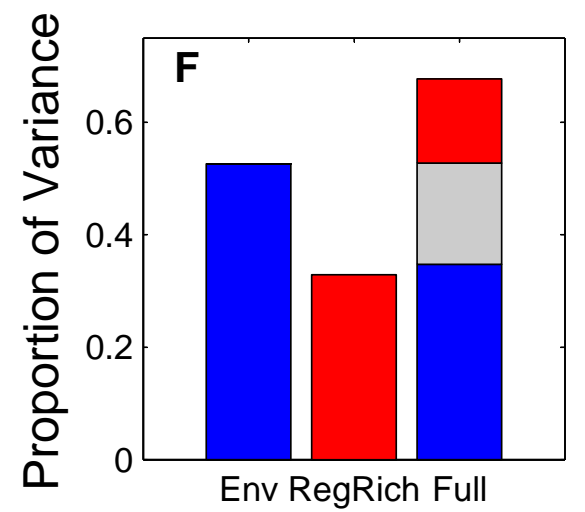




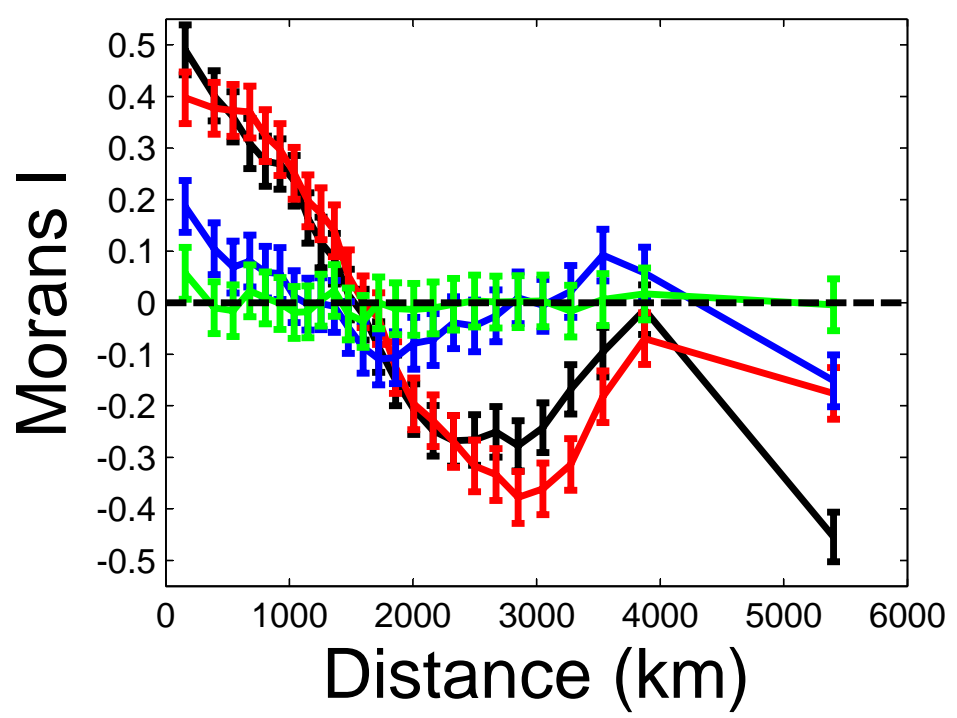




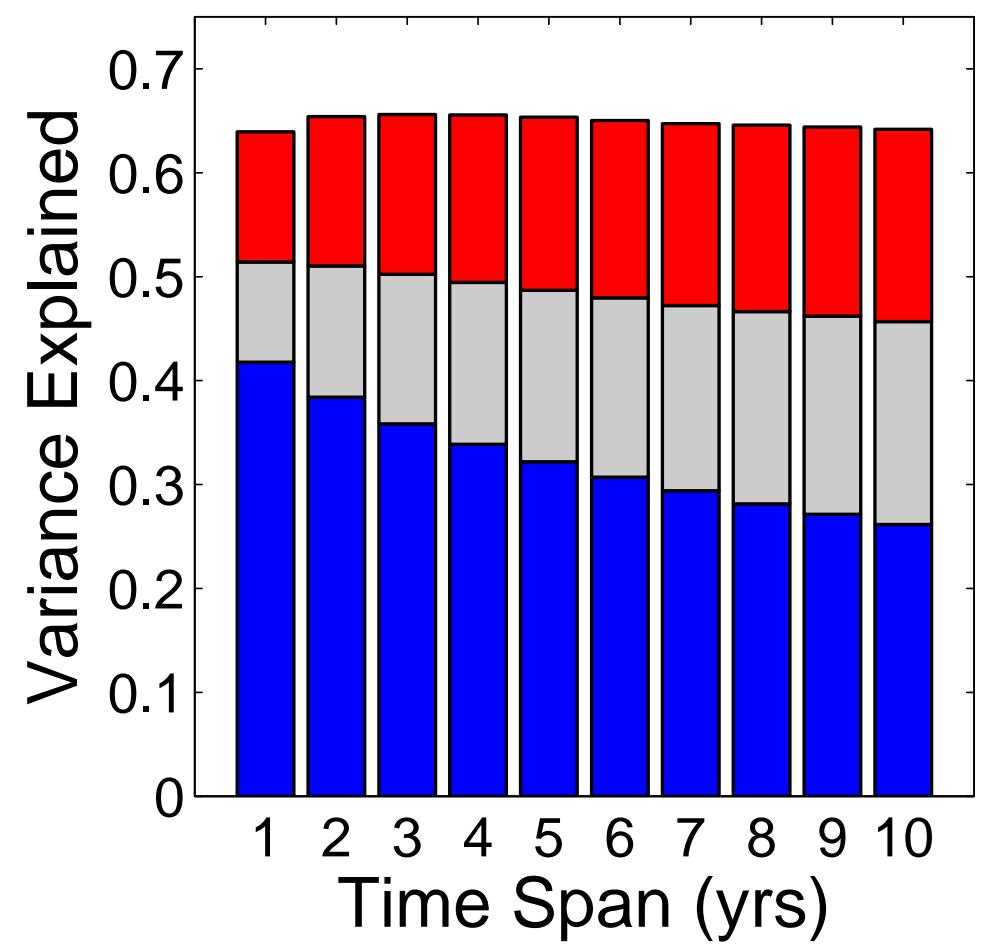





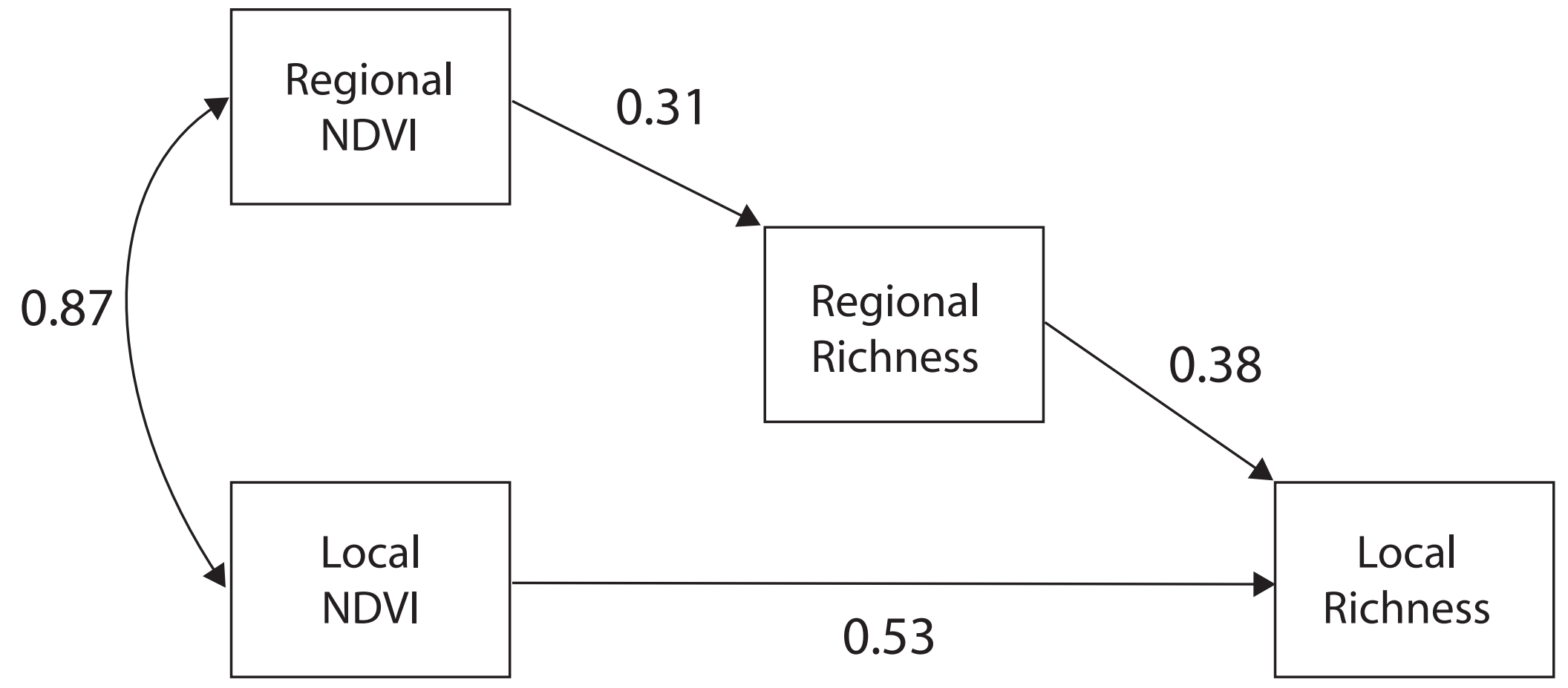

